\title{
SUPERVIVENCIA Y CRECIMIENTO INICIAL DE Parapiptadenia rigida EN CAMPO
}

\author{
Ezequiel Gasparin ${ }^{1 *}$, Maristela Machado Araujo ${ }^{1}$, Thairini Claudino Zavistanovicz ${ }^{2}$, Suelen Carpenedo Aimi ${ }^{2}$, \\ Enrique Benítez León ${ }^{3}$, Álvaro Luís Pasquetti Berghetti ${ }^{2}$ \\ ${ }^{1}$ Universidade Federal de Santa Maria, Departamento de Ciências Florestais, Santa Maria, RS, Brasil - ezequiel_gasparin@hotmail.com \\ (*Autor para correspondência); araujo.maristela@gmail.com. \\ ${ }^{2}$ Universidade Federal de Santa Maria, Programa de Pós-Graduação em Engenharia Florestal, Santa Maria, RS, Brasil - \\ thairini.z@gmail.com; su.aimi@gmail.com; alvaro.berghetti@gmail.com. \\ 3 Universidad Nacional de Asunción. San Lorenzo, Paraguay. - enriqueleon @ hotmail.com.
}

Recebido para publicação: 01/08/2017 - Aceito para publicação: 06/10/2017

\begin{abstract}
Resumen
El presente estudio tuvo como objetivo evaluar el crecimiento de plantas de Parapiptadenia rigida, producidas en vivero con diferentes volúmenes de contenedor y dosis de fertilizante de liberación controlada (FLC), y su crecimiento posterior al trasplante en campo. Las plantas fueron producidas utilizando los siguientes factores: tres volúmenes de contenedor 50, 110 y $180 \mathrm{~cm}^{3}$ y cinco dosis de FLC: 0 (testigo), 3, 6, 9 y $12 \mathrm{~g} \mathrm{~L}^{-1}$ de sustrato, en diseño completamente aleatorio, con cuatro repeticiones. A los 210 días posterior a la siembra, se verificó la altura (H), diámetro de cuello (DC) y relación H/DC de las plantas. Posterior a esa evaluación, se realizó el trasplante a campo, siguiendo los mismos tratamientos de vivero, pero en bloques al azar. Pasados 30 días de la implantación del experimento en campo se evaluó el porcentaje de supervivencia de las plantas, y a los 300 días se verificó el incremento en altura (IH) y diámetro del cuello (IDC); y la relación IH/IDC. Para la producción de plantas en vivero, el contenedor de $180 \mathrm{~cm}^{3}$ proporcionó elevado crecimiento, siendo dependiente de la dosis de FLC utilizada, la cual debe estar entre 6 y $9 \mathrm{~g} \mathrm{~L}^{-1}$. Los resultados obtenidos en vivero se confirmaron en campo para el contenedor utilizado, con mayor supervivencia e incremento para el tubete de $180 \mathrm{~cm}^{3}$. Para la dosis de fertilización, los resultados obtenidos en la producción de plantas se reflejaron solamente en la supervivencia inicial.

Palabras-clave: especie forestal nativa, fertilizante de liberación controlada, volumen de contenedor.
\end{abstract}

\section{Resumo}

Sobrevivência e crescimento inicial de Parapiptadenia rigida a campo. O presente estudo teve como objetivo avaliar o crescimento de mudas de Parapiptadenia rigida, produzidas no viveiro com diferentes volumes de recipientes e doses de fertilizante de liberação controlada (FLC), e seu crescimento após plantio no campo. As mudas foram produzidas utilizando os seguintes fatores: três volumes de recipientes 50,110 e $180 \mathrm{~cm}^{3} \mathrm{e}$ cinco doses de FLC: 0 (testemunha), 3, 6, 9, e $12 \mathrm{~g} \mathrm{~L}^{-1}$ de substrato, em delineamento inteiramente casualizado, com quatro repetições. Aos 210 dias após a semeadura, verificou-se a altura $(\mathrm{H})$, diâmetro do coleto (DC) e relação H/DC das mudas. Posterior a essa avaliação, ocorreu o plantio dessas mudas a campo, seguindo os mesmos tratamentos de viveiro, em blocos ao acaso. Após 30 dias da implantação do experimento no campo avaliou-se a percentagem de sobrevivência das mudas, e aos 300 dias verificou-se o incremento em altura (IH) e diâmetro do coleto (IDC); e a relação IH/IDC. Para a produção das mudas em viveiro, o recipiente de $180 \mathrm{~cm}^{3}$ proporcionou elevado crescimento, sendo dependente da dose de FLC utilizada, a qual deve estar entre 6 e $9 \mathrm{~g} \mathrm{~L}^{-1}$. Os resultados obtidos em viveiro se confirmaram a campo para o recipiente testado, com maiores sobrevivência e incremento para o tubete de $180 \mathrm{~cm}^{3}$. Por outro lado, para a dose de adubação, os resultados obtidos na produção de mudas se refletiram apenas na sobrevivência inicial. Palavras-chave: espécie florestal nativa, fertilizante de liberação controlada, volume de recipiente.

\section{INTRODUCCIÓN}

El aumento de la demanda de productos forestales obtenidos de especies nativas y exóticas, así como el aprovechamiento de esos recursos, lleva a la necesidad de aumentar el área ocupada por masas boscosas, con el fin de abastecer al mercado consumidor. Por lo tanto, el establecimiento de plantaciones forestales, sea para fines comerciales o de restauración, depende de varios factores, siendo la calidad de las plantas un factor fundamental para la supervivencia y el éxito de la plantación (GASPARIN et al., 2014). Así, en los viveros forestales, se busca la utilización de materiales genéticos adaptados al local de la plantación y la aplicación de técnicas silviculturales adecuadas durante el cultivo de cada especie (DAVIS; JACOBS, 2005).

FLORESTA, Curitiba, PR, v. 47, n. 4, p. 533 - 541, out. / dez. 2017.

Gasparin, E. et al.

ISSN eletrônico 1982-4688

DOI: $10.5380 /$ rf.v47i4.54234 
Entre los insumos pasibles de manejo en el proceso de producción de plantas, que pueden favorecer el crecimiento de las mismas, están el tipo de contenedor y la fertilización. La selección del tipo de contenedor, además de influir sobre la cantidad de agua y sustrato disponibles para el crecimiento de la planta, pueden inclusive interferir en aspectos operacionales del proceso productivo, como la disposición de las plantas en el vivero y la mano de obra (LUNA et al., 2009).

Además de eso, Salvador et al. (2004), resaltan que la fertilización es una práctica de rutina utilizada en la producción de plantas en vivero, la cual determina los atributos funcionales de la misma. En ese sentido, el uso de fertilizante de liberación controlada está siendo ampliamente empleado en viveros debido a sus beneficios, entre los que se incluyen la mejora en la nutrición de las plantas, eficiencia del fertilizante y la reducción de mano de obra (KHADDURI, 2014; SILVA et al., 2014). No obstante, las respuestas abordando calidad de plantas, comúnmente son obtenidas en el vivero sin que sean confirmadas en el campo. Este hecho puede conducir a conclusiones precipitadas, trayendo riesgos o perjuicios en el post plantío.

La especie Parapiptadenia rigida (Benth.) Brenan (Fabaceae), presenta una amplia distribución en el Sur de América del Sur (Sur de Brasil, Argentina, Paraguay y Uruguay) (TAULÉ et al., 2012). Posee madera con alta densidad (750 a $1000 \mathrm{Kg} \mathrm{m}^{-3}$ ) y durabilidad, pudiendo ser empleada en la construcción civil y carpintería, siendo, por lo tanto, una especie forestal de gran interés económico (BACKES; IRGANG, 2009). Por otra parte, a pesar de que existen estudios sobre el análisis de semillas y producción de plantas de Parapiptadenia rigida (SCHUMACHER et al., 2004, GASPARIN et al., 2013), poco se conoce sobre su crecimiento inicial en el campo.

De esta forma, se espera que el contenedor de mayor volumen determinará un elevado crecimiento de las plantas en el vivero, independiente de la dosis de fertilización utilizada, y que esos resultados se confirmaran con la plantación en el campo. El presente estudio tuvo como objetivo evaluar el crecimiento de plantas de Parapiptadenia rigida, producidas en vivero con diferentes volúmenes de contenedor y dosis de fertilizante de liberación controlada, y su crecimiento después del trasplante en campo.

\section{MATERIALES Y MÉTODOS}

\section{Producción de plantas}

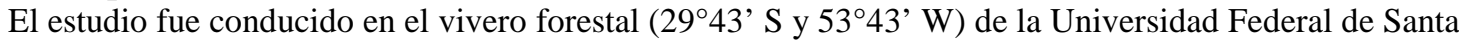
Maria (UFSM), en el Sur de Brasil. La región presenta clima del tipo Cfa (Subtropical húmedo), caracterizado por presentar temperatura media anual variando entre $18^{\circ} \mathrm{C}$ a $20^{\circ} \mathrm{C}$, con lluvias bien distribuidas a lo largo del año, y precipitación media mensual entre 100 y 170 mm (ALVARES et al., 2013).

El experimento fue realizado en dos etapas: producción en vivero y plantación en campo. En el vivero, el experimento fue conducido con un diseño completamente aleatorio, en esquema factorial con cuatro repeticiones, considerando tres volúmenes de contenedor y cinco dosis de fertilizante de liberación controlada (FLC) MiniPril (Osmocote ${ }^{\circledR}$ NPK: 18-05-09) (27 plantas por repetición). Los contenedores utilizados fueron del tipo tubete de polipropileno con los siguientes volúmenes: $50 \mathrm{~cm}^{3}$ (4 estrías, diámetro interno de $26 \mathrm{~mm}$ y altura de $12,5 \mathrm{~cm}), 110 \mathrm{~cm}^{3}$ (6 estrías, diámetro interno de $35 \mathrm{~mm}$ y altura de $\left.13,5 \mathrm{~cm}\right)$ e $180 \mathrm{~cm}^{3}(8$ estrías, diámetro interno de $52 \mathrm{~mm}$ y altura de $13 \mathrm{~cm}$ ); y las dosis de FLC: 0 (testigo), 3, 6, 9, e $12 \mathrm{~g} \mathrm{~L}^{-1}$ de sustrato. Los tubetes fueron llenados con sustrato comercial Carolina Soil ${ }^{\circledR}$, compuesto de turba "Sphagnum" y vermiculita expandida, mezclado con $20 \%$ de cáscara de arroz carbonizada. Además, el sustrato poseía calcáreo dolomítico y yeso agrícola. Dosis de FLC, correspondiente a cada tratamiento, fueron incorporados al sustrato.

Al instalar el experimento, fueron colocadas dos semillas por contenedor. A los 30 días después de la siembra (DDS) fue efectuado el raleo, con el objetivo de eliminar la plántula excedente y posterior intercalado de los contenedores en las bandejas, utilizado el $50 \%$ del área de la bandeja. A los 90 días DDS, se iniciaron las fertilizaciones de cobertura, aplicando fertilizante Peter's Professional ${ }^{\circledR}$ (9-45-15) en la dosis de $3 \mathrm{~g} \mathrm{~L}^{-1}$, semanalmente, vía solución acuosa. Fueron aplicados, aproximadamente, 5 L de la solución en 1.620 plantas.

Las unidades experimentales permanecieron en casa de vegetación por 180 días y, posteriormente, fueron transferidas para el área de pleno sol por más 30 días. Al final de ese periodo, 210 DDS, fueron evaluados la altura de la parte aérea de las plantas $(\mathrm{H})$, con regla graduada $(\mathrm{cm})$, y el diámetro del cuello (DC), con un calibre digital $(\mathrm{mm})$.

\section{Trasplante a campo}

Posterior a la culminación del experimento en vivero, las plantas provenientes de los tratamientos, combinando volúmenes de contenedor y dosis de FLC, fueron plantadas en un área a pleno sol adyacente al vivero. Previamente, fue aplicado herbicida sistémico no selectivo (Glifosato ${ }^{\circledR}$ ) en el total del área y, luego, fueron abiertos pozos circulares de $75 \mathrm{~cm}$ de profundidad y $225 \mathrm{~cm}^{2}$ de área $\left(0,05 \mathrm{~m}^{3}\right)$, con la ayuda de un 
perforador de suelo acoplado a la toma de fuerza de un tractor. Los pozos fueron llenados con tierra de subsuelo, caracterizado por medio de análisis químico realizado en el Laboratorio de Análisis de Suelos de la UFSM, vinculado al CQFS-RS/SC, de acuerdo con Tedesco et al. (1995), conforme observado en el tabla 1.

Tabla 1. Propiedades químicas del subsuelo utilizado para el llenado de los pozos, en la plantación de Parapiptadenia rigida en campo, Santa Maria (RS).

Tabela 1. Propriedades químicas do subsolo utilizado para o preenchimento das covas, no plantio de Parapiptadenia rigida a campo, Santa Maria (RS).

\begin{tabular}{|c|c|c|c|c|c|c|c|c|c|c|c|}
\hline $\begin{array}{c}\mathrm{pH} \\
\left(\mathrm{H}_{2} \mathrm{O}\right)\end{array}$ & $\begin{array}{l}\text { MO } \\
(\%)\end{array}$ & $\mathrm{P}_{\text {-Mehlich }}$ & $\mathrm{K}$ & $\mathrm{CTC}_{\mathrm{pH} 7,0}$ & $\mathrm{Ca}$ & $\mathrm{Mg}$ & $\mathrm{Al}$ & $\mathrm{H}+\mathrm{Al}$ & CTC ${ }_{\text {efet. }}$ & \multirow[t]{2}{*}{$\begin{array}{l}\text { Índice } \\
\text { SMP }\end{array}$} & \multirow[t]{2}{*}{ Textura } \\
\hline $1: 1$ & $\mathrm{~m} / \mathrm{v}$ & \multicolumn{2}{|c|}{$\mathrm{mg} \mathrm{dm}^{-3}$} & & & \multicolumn{4}{|c|}{$\mathrm{cmol}_{\mathrm{c}} \mathrm{dm}^{-3}$} & & \\
\hline 4,6 & 3,0 & 9,3 & 92 & 15,3 & 5,6 & 1,7 & 0,7 & 7,7 & 8,3 & 5,5 & 3,0 \\
\hline
\end{tabular}

El experimento fue instalado en diseño de bloques al azar, con cuatro repeticiones y cuatro plantas por parcela, con espaciamiento de $1 \mathrm{~m}$ x $1 \mathrm{~m}$, en esquema factorial (tres volúmenes de contenedor x cinco dosis de FLC), totalizando 240 plantas. En el primer mes posterior al trasplante, fueron efectuados riegos semanales, con aproximadamente, $1 \mathrm{~L}$ de agua por planta, utilizando una regadera. Las fertilizaciones de cobertura fueron realizadas a los 30 y 240 días posterior al trasplante en todos los tratamientos, utilizando $100 \mathrm{~g}$ de fertilizante por planta (NPK 5-20-20).

El control de hormigas cortadoras fue realizado en todo el transcurso del experimento, por medio de la aplicación de hormiguicida granulado a base de sulfluramida, cuando fue necesario. También se realizó el control de malezas con el coronado y aplicación de herbicida alrededor de las plantas, con un pulverizador tipo mochila.

Treinta días después de la instalación del experimento se verificó el porcentaje de soupervivencia de las plantas (\%), y a los 300 días después del trasplante (DDT) fueron medidos la altura (H) y el diámetro del cuello DC, con la finalidad de efectuar el cálculo del incremento en altura (IH) y el incremento en diámetro del cuello (IDC), substrayendo los valores medidos a los 300 días de aquellos medidos antes del trasplante (210 DDS).

\section{Análisis estadístico}

Los datos fueron verificados en cuanto a las presuposiciones de normalidad de la distribución de los errores y la homogeneidad de la varianza, por las pruebas de Shapiro-Wilk y Bartlett, respectivamente, con la utilización del software Action. Posteriormente, los mismos fueron sometidos a análisis de varianza y determinada la significancia de los efectos principales de los factores y de las interacciones. Las medias fueron comparadas por la prueba de Scott-Knott y/o regresión polinomial $(\alpha 0,05)$. Cuando fue posible, se calculó la dosis de máxima eficiencia técnica (DMET), por medio de la fórmula: $X=-b_{1} / 2 b_{2}$ en que: $X=$ punto de máxima eficiencia técnica; $b_{1}$ y $b_{2}=$ coeficientes de la ecuación. Para los análisis se utilizó el software estadístico Sisvar (FERREIRA, 2011). Debido a la elevada mortalidad observada en el tratamiento testigo (dosis $0 \mathrm{~g} \mathrm{~L}^{-1} \mathrm{de}$ FLC) para los tres volúmenes de contenedor, esos tratamientos fueron desconsiderados del análisis de los datos de plantación a campo.

\section{RESULTADOS}

Al final del ciclo de producción de las plantas de Parapiptadenia rigida en vivero (210 DDS), se verificó que, para $\mathrm{H}$, hubo interacción entre los volúmenes de contenedor y las dosis de FLC (figura 1). En todas las dosis probadas, los valores más elevados de altura fueron constatados en el contenedor de mayor volumen $\left(180 \mathrm{~cm}^{3}\right)$, DMET de $9,38 \mathrm{~g} \mathrm{~L}^{-1}$. Para todas las dosis utilizadas, ese contenedor, se mostró significativamente superior al de $50 \mathrm{~cm}^{3}$. Se destaca que los menores valores de altura fueron observados en los tratamientos sin fertilización, sin importar el volumen de contenedor, alcanzando altura máxima de $3,8 \mathrm{~cm}$. 


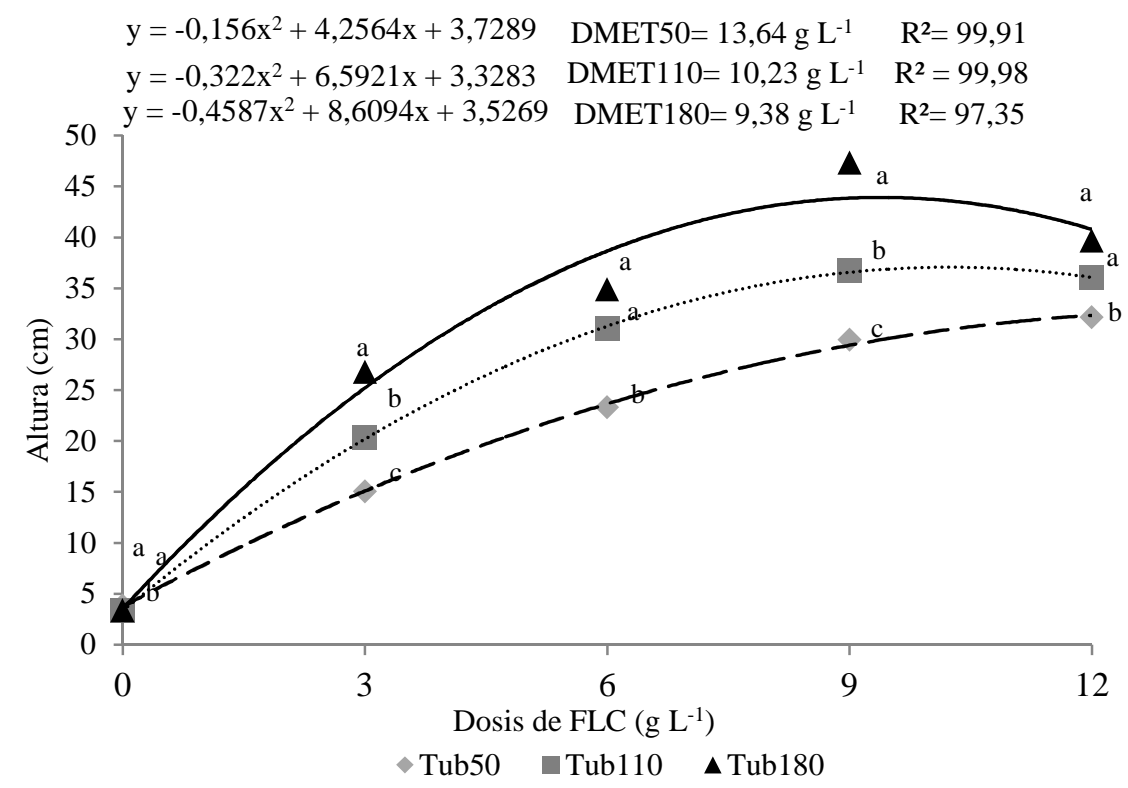

Figura 1. Altura (cm) de plantas de Parapiptadenia rigida, producidas en diferentes volúmenes de contenedor y dosis de fertilizante de liberación controlada (FLC), a los 210 días en vivero.

Figura 1. Altura $(\mathrm{cm})$ de plantas de Parapiptadenia rigida, produzidas em diferentes volumes de recipiente e doses de fertilizante de liberação controlada (FLC), aos 210 dias em viveiro.

Para el diámetro del cuello, hubo diferencia significativa en ambos factores estudiados $(p<0,05)$. En relación al volumen de contenedor utilizado, el uso del tubete de $180 \mathrm{~cm}^{3}$ posibilitó mayor crecimiento del diámetro del cuello $(3,41 \mathrm{~mm})$, difiriendo significativamente de los volúmenes de 110 y $50 \mathrm{~cm}^{3}(3,27$ y $3,18 \mathrm{~mm}$, respectivamente) (tabla 2 ).

Tabla 2. Diámetro del cuello (DC 210) de las plantas de Parapiptadenia rigida producidas en diferentes volúmenes de contenedor, a los 210 días en vivero; supervivencia (S\%) de las plantas 30 días después del trasplante en campo; incremento en altura (IH), incremento en diámetro del cuello (IDC) y relación altura y diámetro del cuello (H/DC), 300 días después del trasplante en campo.

Tabela 2. Diâmetro do coleto (DC 210) de plantas de Parapiptadenia rigida produzidas em diferentes volumes de recipiente, aos 210 dias em viveiro; sobrevivência (S\%) das plantas 30 dias após o plantio a campo; incremento em altura (IH), incremento em diâmetro do coleto (IDC) e relação altura e diâmetro do coleto (H/DC), 300 dias após o plantio a campo.

\begin{tabular}{cccccc}
\hline Volumen de contenedor $\left(\mathbf{c m}^{3}\right)$ & DC 210 (mm) & S (\%) & IH $(\mathbf{c m})$ & IDC $(\mathbf{m m})$ & H/DC \\
\hline 50 & $3,18 \mathrm{~b}^{*}$ & $61 \mathrm{~b}$ & $80,63 \mathrm{~b}$ & $13,88 \mathrm{~b}$ & $6,04 \mathrm{a}$ \\
110 & $3,27 \mathrm{~b}$ & $71 \mathrm{a}$ & $77,46 \mathrm{~b}$ & $14,67 \mathrm{~b}$ & $5,87 \mathrm{~b}$ \\
180 & $3,41 \mathrm{a}$ & $79 \mathrm{a}$ & $111,33 \mathrm{a}$ & $18,00 \mathrm{a}$ & $6,77 \mathrm{a}$ \\
\hline
\end{tabular}

* Medias seguidas de la misma letra en la columna no difieren entre sí por el Test de Scott-Knott $(p \leq 0,05)$; ns= no significativo.

Para el factor dosis de fertilizante de liberación controlada, el diámetro del cuello difirió significativamente entre las mismas, presentando comportamiento cuadrático, siendo la DMET verificada en $9,25 \mathrm{~g} \mathrm{~L}^{-1}$, para 4,29 mm de diámetro del cuello (figura 2). 


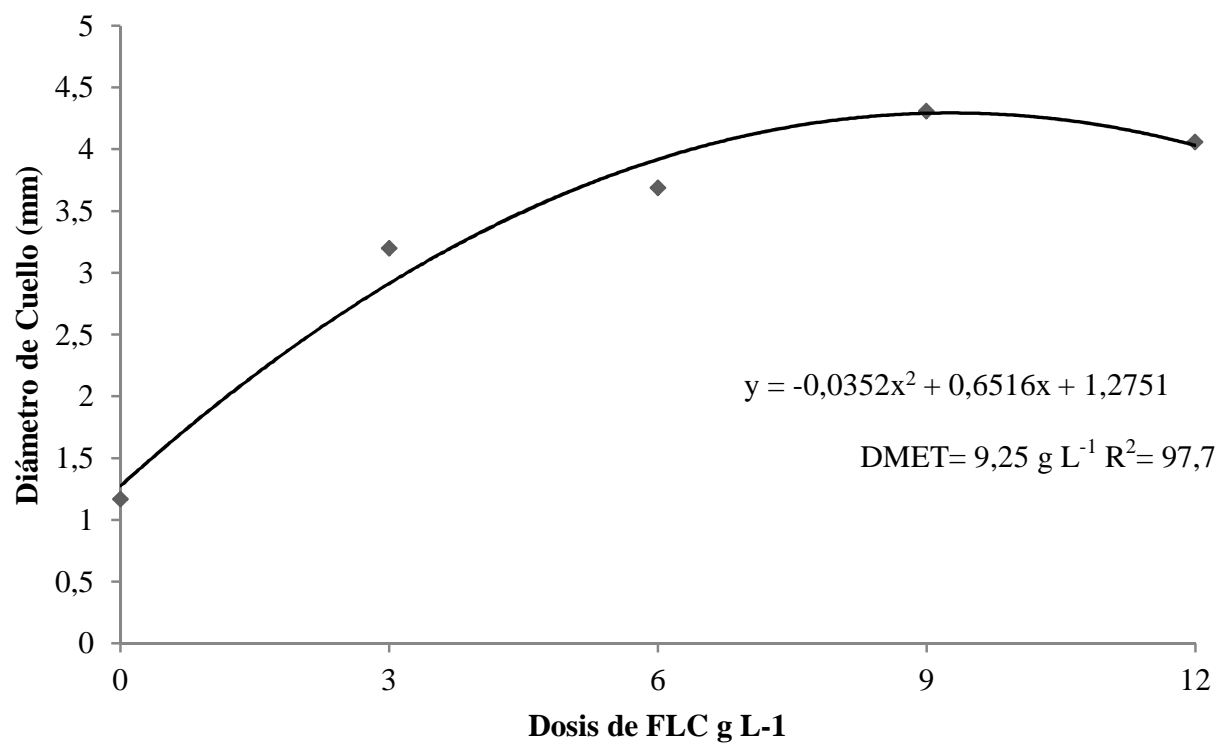

Figura 2. Diámetro del cuello (DC) de plantas de Parapiptadenia rigida, producidas con diferentes dosis de fertilizante de liberación controlada (FLC), a los 210 días en vivero.

Figura 2. Diâmetro do coleto (DC) de plantas de Parapiptadenia rigida plants, produzidas em diferentes volumes de recipiente e doses de fertilizante de liberação controlada (FLC), aos 210 dias em viveiro.

En relación al análisis de los datos de la plantación en campo, se verificó elevada mortalidad inicial $(83 \%)$ de las plantas en los tratamientos que correspondieron al testigo (sin fertilización), independiente del volumen de contenedor utilizado, motivo por el que se optó no incluirlos en el análisis estadístico.

De acuerdo con los resultados obtenidos para la supervivencia inicial de las plantas, desconsiderándose los tratamientos testigo, se observó diferencia significativa para los factores independientes $(p<0,05)$. Las plantas producidas en los tubetes de 110 y $180 \mathrm{~cm}^{3}$ no difirieron entre sí, presentando valores de 71 y $79 \%$, respectivamente, mientras aquellas producidas en tubetes de $50 \mathrm{~cm}^{3}$ tuvieron menor supervivencia (61\%) (tabla 2). En cuanto a las dosis de FLC, se verificó comportamiento cuadrático, con DMET correspondiendo a $7,7 \mathrm{~g} \mathrm{~L}^{-1}$ (figura 3) y menor supervivencia de las plantas.

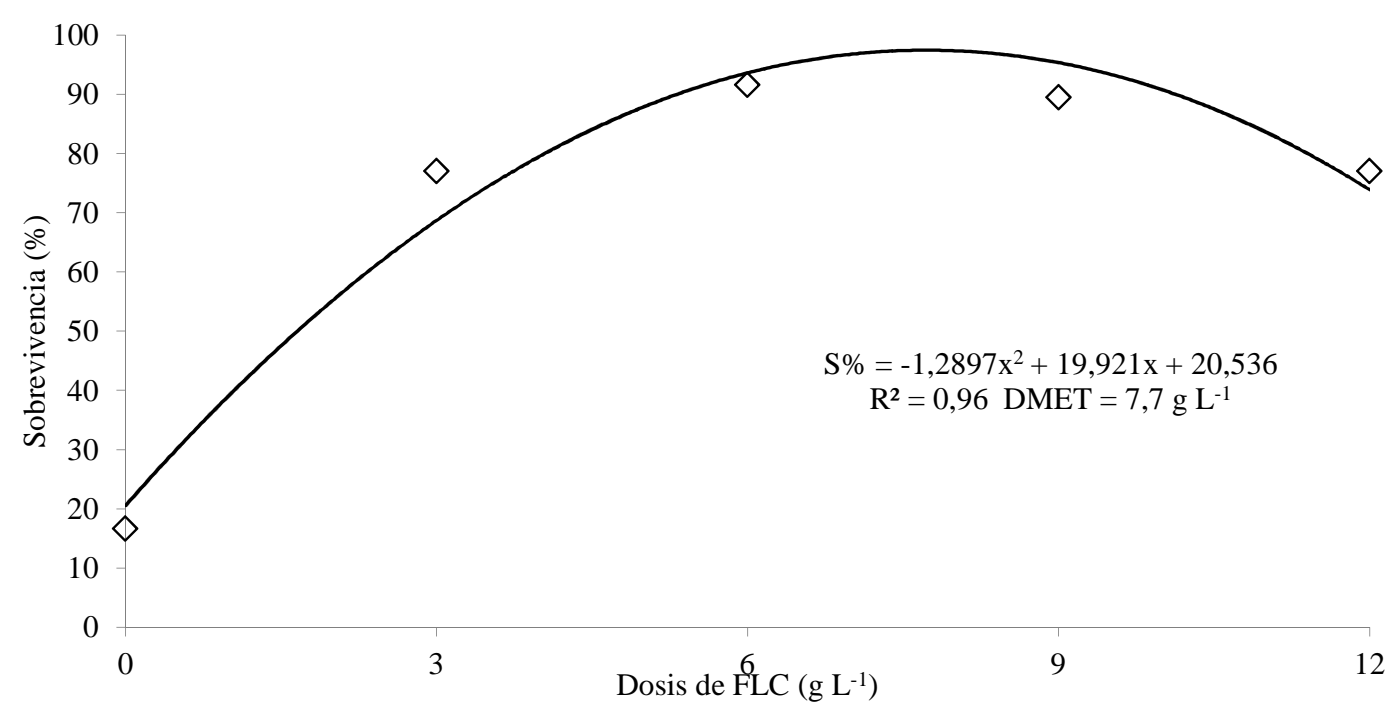

Figura 3. Tasa de supervivencia (S\%) de plantas de Parapiptadenia rigida, 30 días después del trasplante en campo, en función de las dosis de fertilizante de liberación controlada (FLC).

FLORESTA, Curitiba, PR, v. 47, n. 4, p. 533 - 541, out. / dez. 2017.

Gasparin, E. et al.

ISSN eletrônico 1982-4688

DOI: $10.5380 /$ rf.v47i4.54234 
Figura 3. Taxa de sobrevivência (S\%) de plantas de Parapiptadenia rigida, 30 dias após plantio a campo, em função das doses de fertilizante de liberação controlada (FLC).

En relación a las variables incremento en altura (IH) e incremento en diámetro del cuello (IDC), 300 días después del trasplante en campo, se verificó diferencia significativa entre volúmenes de contenedor. Esas variables presentaron comportamientos semejantes entre sí, con medias superiores de IH e IDC de $111,33 \mathrm{~cm} \mathrm{y}$ $18,00 \mathrm{~mm}$, respectivamente, para el tubete de $180 \mathrm{~cm}^{3}$ (tabla 2).

En relación a las dosis de FLC, no hubo diferencia significativa $(p>0,05)$ para las variables IH e IDC, inclusive con la menor dosis utilizada $\left(3 \mathrm{~g} \mathrm{~L}^{-1}\right)$, siendo que la media general para este factor fue de $89,81 \mathrm{~cm}$ para IH y $15,52 \mathrm{~mm}$ para IDC.

\section{DISCUSIÓN}

Algunas investigaciones relatan la importancia de evaluar el comportamiento de las plantas en vivero, aliado a su crecimiento en campo (MATTSON, 1996, SALVADOR et al. 2004, LERENA et al. 2006, ZIDA et al. 2008). Esos estudios identifican las variables y condicionantes que pueden influir en los estadios de crecimiento de las plantas, teniendo en cuenta que algunos factores que interfieren en la fase de vivero pueden o no ser los mismos que determinaran el comportamiento de las plantas posterior a su trasplante.

Se evidenció que el mayor volumen de tubete utilizado $\left(180 \mathrm{~cm}^{3}\right)$ proporcionó elevado crecimiento de las plantas en la fase de vivero. Tal hecho ocurrió debido a que contenedores con mayor volumen soportan mayor cantidad de sustrato, favoreciendo la disponibilidad de agua y nutrientes, posibilitando un adecuado desarrollo del sistema radical (LERENA et al. 2006). Además, por controlar la cantidad de raíces que la planta puede producir, el volumen del contenedor determina la altura de la parte aérea a lo largo de un ciclo de producción (RITCHIE et al. 2010).

Resultados semejantes fueron relatados por Ferraz y Engel (2011) para plantas de Parapiptadenia rigida en vivero, al constatar que la especie es altamente sensible a la variación del volumen de contenedores, presentando elevado crecimiento, tanto en la parte aérea como en la parte radical para el contenedor de mayor volumen $\left(300 \mathrm{~cm}^{3}\right)$, indicando así, la exigencia de esa especie en relación a un mayor espacio para expandir su sistema radical.

Muchas veces, se opta por el uso de contenedores con menor volumen debido a la economía del sustrato, fertilizantes y área ocupada del vivero, no obstante, Luna et al. (2009) y Poorter et al. (2012) resaltan que los beneficios de la selección de contenedores mayores pueden acabar compensando su costo, por ejemplo, obteniendo mayor supervivencia y crecimiento de las plantas en el campo, como fue constatado en el presente trabajo.

Además, se verificó que el uso de tubetes con menor volumen $\left(50\right.$ y $\left.110 \mathrm{~cm}^{3}\right)$ ocasionó restricciones al crecimiento de las plantas en el vivero. Contenedores menores que lo necesario acaban impidiendo el crecimiento radical (POORTER et al. 2012), y consecuentemente, de la parte aérea. Por lo tanto, tales volúmenes de contenedores deben ser evitados en la producción de Parapiptadenia rigida, ya que su utilización podrá implicar plantas de baja calidad.

En relación a las dosis de fertilización utilizadas en la producción de plantas, se verificó que el uso de $9 \mathrm{~g}$ $\mathrm{L}^{-1}$ de FLC es capaz de proporcionar el mayor crecimiento, tanto en altura como en diámetro del cuello. Eso se explica por el hecho de que el estado nutricional de las plantas influye en el crecimiento y el desempeño inicial en el campo (GROSSNICKLE et al. 2012), siendo que la deficiencia de nutrientes arrastra problemas fisiológicos en la planta, como disminución de la actividad fotosintética, resultando en baja acumulación de biomasa (ZHAO et al. 2005, YONG et al. 2010), tornando a las plantas susceptibles a las adversidades del ambiente, como ataque de plagas y déficit hídrico, siendo imprescindible la fertilización en la fase inicial de la producción.

Viveros que producen especies forestales, comúnmente utilizan dosificaciones mínimas de fertilización que promueven su adecuado crecimiento. Muchas veces esto puede favorecer una limitación en el almacenamiento de nutrientes por las plantas, los cuales podrían ser utilizados posteriormente y favorecer la supervivencia y crecimiento inicial (HAASE et al. 2006, SALIFU et al. 2009).

En cuanto al crecimiento de las plantas de Parapiptadenia rigida en el campo, el mayor volumen de tubete $\left(180 \mathrm{~cm}^{3}\right)$ proporcionó alta supervivencia y crecimiento en la parte aérea. La supervivencia inicial de las plantas está directamente relacionada con el volumen del contenedor, considerando que según Ritchie et al. (2010), el sistema radical de plantas producidas en contenedores mayores posee amplia superficie de contacto con el suelo circundante cuando son llevadas al campo, facilitando la absorción de agua y nutrientes, los cuales son esenciales para su supervivencia y crecimiento.

Se verificó por medio de la relación H/DC, que las plantas producidas en contenedores de 50 y $180 \mathrm{~cm}^{3}$ presentaron mayor incremento en altura que de diámetro del cuello, cuando son comparadas con aquellas 
producidas en los tubetes de $110 \mathrm{~cm}^{3}$. La relación H/DC busca demostrar la proporción de resistencia de las plantas a la acción de factores estresantes, como viento y competición con malezas, siendo que valores bajos de H/DC pueden indicar plantas con alta resistencia (RITCHIE et al. 2010). Por otra parte, en este estudio, se verifica que a pesar de la mayor relación obtenida $\left(6,77\right.$ en tubetes de $\left.180 \mathrm{~cm}^{3}\right)$, las plantas respondieron favorablemente, por lo que se puede considerar como aceptable dicho valor de H/DC para esta especie.

Fue posible observar, inclusive, la alta mortalidad en el campo (83\%) de las plantas que no recibieron fertilización de base durante su producción. La disponibilidad de nutrientes favoreció la supervivencia y el incremento, tanto en altura como en diámetro del cuello de las plantas en campo, independiente de la dosis aplicada $\left(3,6,9\right.$ o $\left.12 \mathrm{~g} \mathrm{~L}^{-1}\right)$.

Estos estudios demuestran la importancia de investigar la demanda nutricional para cada especie en toda la fase de producción, pues, como en el presente caso, se verificó que las dosis de fertilización no interfirieron en el incremento en H y DC para Parapiptadenia rigida después de 300 días de plantación. No obstante eso, fueron determinantes para su supervivencia inicial. De esta forma se sugiere priorizar, en la producción de plantas, la aplicación de dosis de FLC que proveerá la mayor tasa de supervivencia en campo, reduciendo los costos de reposición.

\section{CONCLUSIONES}

- La hipótesis de que el contenedor con mayor volumen $\left(180 \mathrm{~cm}^{3}\right)$ proporcionaría un elevado crecimiento de las plantas en vivero fue aceptada, no obstante, el crecimiento fue dependiente de la dosis de FLC utilizada, la cual debe estar entre 6 y $9 \mathrm{~g} \mathrm{~L}^{-1}$.

- Los resultados obtenidos en el vivero se confirmaron en el campo para el contenedor utilizado, obteniendo mayor tasa de supervivencia e incremento en altura y diámetro del cuello para plantas producidas en tubetes de $180 \mathrm{~cm}^{3}$.

- En cuanto a la dosis de fertilización, los resultados de vivero se confirmaron solamente en la supervivencia inicial de las plantas, no reflejándose en su crecimiento.

\section{REFERENCIAS}

ALVARES, C. A.; STAPE, J. L.; SENTELHAS, P. C.; GONÇALVES, J. L. M.; SPAROVEK, G. Köppen's climate classification map for Brazil. Meteorologische Zeitschrift, v. 22, p. 711-728, 2013.

BACKES, P.; IRGANG, B. Árvores do sul: guia de identificação e interesse ecológico. Porto Alegre: Paisagem do Sul, 2009, 332 p.

DAVIS, A. S.; JACOBS, D. F. Quantifying root system quality of nursery seedlings and relationship to outplanting performance. New Forests, v. 30, p. 295-311, 2005.

FERRAZ, A. V.; ENGEL, V. L. Efeito do tamanho de tubetes na qualidade de mudas de jatobá (Hymenaea courbaril 1. var. stilbocarpa (Hayne) Lee et Lang.), ipê-amarelo (Tabebuia chrysotricha (Mart. Ex DC.) Sandl.) e guarucaia (Parapiptadenia rigida (Benth.) Brenan). Revista Árvore, v. 35, n. 3, p. 413-423, 2011.

FERREIRA, D. F. Sisvar: a computer statistical analysis system. Ciência e Agrotecnologia v. 35, n. 6, p. 10391042, 2011.

GASPARIN, E.; ARAUJO, M. M.; TOLFO, C. V.; FOLTZ, D. R. B.; MAGISTRALI, P. R. Substrates for germination and physiological quality of storage seeds of Parapiptadenia rigida (Benth.) Brenan. Journal of Seed Science, v. 35, n. 1, p. 77-85, 2013.

GASPARIN, E.; DE AVILA, A. L.; ARAUJO, M. M.; CARGNELUTTI, A. F.; DORNELES, D. U.; FOLTZ, D. R. B. Influência do substrato e do volume de recipiente na qualidade das mudas de Cabralea canjerana (vell.) mart. em viveiro e no campo. Ciência Florestal, v. 24, n. 3, p. 553-563, 2014.

GROSSNICKLE, S. C. Why seedlings survive: influence of plant attributes. New Forests, v. 43, p. 711-738, 2012.

HAASE, D.; ROSE, R. TROBAUGHT, J. Field performance of three stock sizes of Douglas-fir container seedlings grown with slow-release fertilizer in the nursery growing medium. New Forests, v. 31, p. 1-24, 2006.

KHADDURI, N. Benefits of small-prill controlled-release fertilizer in container production. In: WILKINSON, K. M.; HAASE, D. L.; PINTO, J. R. National Proceedings: Forest and Conservation Nursery Associations-

FLORESTA, Curitiba, PR, v. 47, n. 4, p. 533 - 541, out. / dez. 2017.

Gasparin, E. et al.

ISSN eletrônico 1982-4688

DOI: $10.5380 /$ rf.v47i4.54234 
2013. Fort Collins (CO): USDA Forest Service, Rocky Mountain Research Station. Proceedings RMRS-P-72. p. 54-56, 2014.

LERENA, S. D.; SIERRA, N. H.; MANZANO, I. C.; BUENO, L. O.; RUBIRA, J. L. P.; MEXAL, J. G.. Container characteristics influence Pinus pinea seedling development in the nursery and field. Forest Ecology and Management, v. 221, p. 63-71, 2006.

LUNA, T.; LANDIS, T. D.; DUMROESE, R. K. Containers. In: DUMROESE, R. K.; LUNA, T.; LANDIS, T. D. Nursery manual for native plants: a guide for tribal nurseries. Nursery management. Agriculture Handbook 730. Washington. D.C.: U.S. Department of Agriculture, Forest Service, v. 1, p. 95-111, 2009.

MATTSON, A. Predicting field performance using seedling quality assessment. New Forests, v. 13, p. 223-248, 1996.

POORTER, H.; BÜHLER, J.; DUSSCHOTEN, D.; CLIMENT, J.; POSTMA, J. A. Pot size matters: a metaanalysis of the effects of rooting volume on plant growth. Functional Plant Biology, v. 39, p. 839-850, 2012.

RITCHIE, G. A.; LANDIS, T. D.; DUMROESE, R. K.; HAASE, D. L. Assessing Plant Quality. In: LANDIS, T. D.; DUMROESE, R. K.; HAASE, D. L. The container tree nursery manual: Seedling processing, storage, and outplanting. Agric. Handbook 674. Washington: United States Department of Agriculture Forest Service v. 7, p. 19-81, 2010.

SALIFU, K. F.; JACOBS, D. F.; BIRGE, Z. K. Nursery nitrogen loading improves field performance of Bareroot Oak seedlings planted on abandoned mine lands. Restoration Ecology, v. 17, n. 3, p. 339-349, 2009.

SALVADOR, P. V.; PLANELlES, R.; ENRÍQUEZ, E.; RUBIRA, J. P. Nursery cultivation regimes, plant functional attributes, and field performance relationships in the Mediterranean oak Quercus ilex L. Forest Ecology and Management, v. 196, p. 257-266, 2004.

SCHUMACHER, M. V.; CECONI, D. E.; SANTANA, C. A. Influência de diferentes doses de fósforo no crescimento de mudas de angico-vermelho (Parapiptadenia rigida (bentham) brenan). Revista Árvore, v. 28 , n. 1, p. 149-155, 2004.

SILVA, R. B. G.; SILVA, M. R.; SIMÕES, D. Substrates and controlled-release fertilizations on the quality of eucalyptus cuttings. Revista Brasileira de Engenharia Agrícola e Ambiental, v. 18, n. 11, p. 1124-1129, 2014.

TAUlÉ, C.; ZABALETA, M.; MAREQUE, C.; PLATERO, R.; SANJURJO, L.; SICARDI, M.; FRIONI, L.; BATTISTONI, F.; FABIANO, E. New Betaproteobacterial Rhizobium strains able to efficiently nodulate Parapiptadenia rigida (Benth.) Brenan. Applied Environmental Microbiology, v. 78, n. 6, p. 1692-1700, 2012.

TEDESCO, M. J.; GIANEllo, C.; BISSANI, C. A.; BOHNEN, H.; VOLKWEISS, S. J. Análise de solo, plantas e outros materiais. 2.ed. Porto Alegre: Universidade Federal do Rio Grande do Sul, 1995.147 p. (Boletim Técnico 5).

YONG, J. W. H.; NG, Y. F.; TAN, S. N.; CHEW, A. Y. L. Effect of fertilizer application on photosynthesis and oil yield of Jatropha curcas L. Photosynthetica, v. 48, n. 2, p. 208-218, 2010.

ZHAO, D.; REDDY, K. R.; KAKANIA, V. G.; REDDY, V. R. Nitrogen deficiency effects on plant growth, leaf photosynthesis, and hyperspectral reflectance properties of sorghum. European journal of agronomy, v. 22, p. 391-403, 2005.

ZIDA, D.; TIGABU, M.; SAWADOGO, L.; ODE`N, P. C. Initial seedling morphological characteristics and field performance of two Sudanian savanna species in relation to nursery production period and watering regimes. Forest Ecology and Management, v. 255, p. 2151-2162, 2008. 
FLORESTA, Curitiba, PR, v. 47, n. 4, p. 533 - 541, out. / dez. 2017.

Gasparin, E. et al.

ISSN eletrônico 1982-4688

DOI: $10.5380 /$ rf.v47i4.54234 